\title{
Ultrasound molecular imaging for differentiation of benign and malignant tumors in patients
}

\author{
Fei Yan $^{1}$, Zhuqing Song ${ }^{2}$, Meng Du ${ }^{3}$, Alexander L. Klibanov ${ }^{4}$ \\ ${ }^{1}$ Paul C. Lauterbur Research Center for Biomedical Imaging, Institute of Biomedical and Health Engineering, Shenzhen Institutes of Advanced \\ Technology, Chinese Academy of Sciences, Shenzhen 518055, China; ${ }^{2}$ Department of Breast Surgery, Peking University Shenzhen Hospital, \\ Shenzhen 518036, China; ${ }^{3}$ Department of Ultrasound Medicine, Laboratory of Ultrasound Molecular Imaging, The Third Affiliated Hospital \\ of Guangzhou Medical University, Guangzhou 510150, China; ${ }^{4}$ Cardiovascular Division and Robert M. Berne Cardiovascular Imaging Center, \\ University of Virginia, Charlottesville, VA, 22908, USA
}

Correspondence to: Fei Yan. Paul C. Lauterbur Research Center for Biomedical Imaging, Institute of Biomedical and Health Engineering, Shenzhen Institutes of Advanced Technology, Chinese Academy of Sciences, Shenzhen 518055, China. Email: fei.yan@siat.ac.cn.

Submitted Nov 20, 2018. Accepted for publication Dec 10, 2018.

doi: 10.21037/qims.2018.12.08

View this article at: http://dx.doi.org/10.21037/qims.2018.12.08

\section{Introduction}

The differentiation of benign and malignant tumors is of great significance for the preliminary evaluation of biological behaviors and prognosis of tumors prior to surgery or other interventions (1). Histopathological examination of the tumor biopsy samples serves as the gold standard for confirming the degree of malignancy of tumors. However, it is desirable and valuable to explore noninvasive imaging approaches to obtain diagnostic information at the earliest possible time points and without biopsy. When compared with other imaging modalities, ultrasound offers many advantages. It is safe, sensitive and portable. It offers a lower cost, yet also provides the features of realtime imaging and deep tissue penetration. Traditional ultrasound imaging technologies include B-mode-based grayscale ultrasonography, blood-flow-based color Doppler flow imaging (CDFI) and stiffness-based elastography. These techniques reflect local anatomy and can provide precursory information on the benign and malignant status of tumors $(2,3)$. For example, the representative grayscale ultrasonography of malignant neoplasms is considered to be hypoechoic in grey scale, with fuzzy boundary often accompanied by microcalcification (4). CDFI is generally used to determine the benign $v s$. malignant tumor status from the blood vessel density and blood flow spectrum data $(5,6)$. Contrast-enhanced ultrasound (CEUS) is capable of assessing the blood flow in the lesions through perfusion imaging of small capillary blood vessels $(7,8)$. To some degree, all these methods provide diagnostic information on the benign or malignant status of the interrogated lesions. However, these ultrasound techniques mostly reflect the characteristics of tumor anatomy.

During the past 20 years, ultrasound elastography has developed as a supplement to traditional ultrasound technology for differentiation of benign and malignant tumors, especially in superficial organs (9). Based on the heterogeneity of stiffness between different tumor tissues, ultrasound elastography can distinguish between benign and malignant tumors by detecting the modulus of elasticity (10). Studies have confirmed that, compared with conventional ultrasonography, ultrasound elastography has better specificity and accuracy in differentiating benign from malignant breast tumors $(11,12)$. Nevertheless, ultrasound elastography has limitations in precise tumor diagnosis. The results of ultrasound elastography were easily affected by the skills of the operators and the location of the lesion, which might cause false positive results and reduce the diagnosis accuracy (13). Therefore, it is very important to develop novel ultrasound imaging technology to acquire functional information, especially the information on the tumor status at the molecular level, which is currently only available from invasive techniques, such as biopsy and histology. Fortunately, ultrasound technology has emerged with an exciting molecular imaging potential in tumor 
diagnosis.

Ultrasound molecular imaging with targeted probes requires specific targeting to the receptors overexpressed on the angiogenic blood vessels. Such targeted contrast agents are administered intravenously, and accumulate in the vasculature in the areas of disease (14). Therefore, microbubbles, the most popular ultrasound contrast agents, need to be functionalized with appropriate ligands that have high affinity to the target vasculature biomarkers of disease, and allow specific binding and retention of these particles on the target vessel surface, despite the dislodging action of blood flow. Numerous targeting ligands, including antibodies, antibody fragments, peptides, and carbohydrates have been applied to construct targeted microbubble probes (15). By detecting the signals derived from the retained microbubbles in the target regions, ultrasound molecular imaging can determine the expression level of molecules and visualize biomarker status in situ. Multiple studies in vivo have demonstrated the diagnostic utility of ultrasound molecular imaging for the detection of inflammation, atherosclerosis and tumor angiogenesis in animal studies (16-19).

\section{Ultrasound molecular imaging in patients}

Recently, Smeenge et al. conducted the first in-human, Phase $0-1$, exploratory study to investigate the feasibility and safety of the ultrasound molecular imaging technology for the detection of prostate cancer in men. This study was performed with BR55 ultrasound contrast, a formulation of microbubbles that are decorated with a heterodimeric peptide which possesses a specific affinity to a kinase insert domain receptor (KDR). Imaging was performed in 24 patients with biopsy-proven prostate cancers scheduled for radical prostatectomy using a clinical ultrasound scanner at low acoustic power. A total of 52 lesions were determined to be malignant by histopathology with $26(50 \%)$ of them observed during BR55 imaging. After fine-tuning BR55 dosing and imaging protocol in the 12 subsequent patients, $19(68 \%)$ in a total of 28 determined malignant lesions were identified during BR55 ultrasound molecular imaging. No serious adverse events or clinically meaningful changes in the physical examination, blood pressure and heart rate measurements, electrocardiogram, and blood sampling data were identified during or after administration (20).

More recently, BR5 5 was also used for ultrasound molecular imaging in a clinical trial in patients with breast and ovarian lesions (Figure 1) (21). In this study, 24 women with ovarian lesions and 21 women with breast lesions were injected intravenously with BR55 and received ultrasound molecular imaging. Seventy-seven percent of malignant ovarian lesions and $93 \%$ of malignant breast lesions exhibited strong KDR-targeted ultrasound molecular imaging signals. No targeted signals were seen in $78 \%$ of benign ovarian lesions and $67 \%$ of benign breast lesions. KDR expression on immunohistochemistry matched well with ultrasound molecular imaging signal in $93 \%$ of breast and $85 \%$ of ovarian malignant lesions. A typical result of ultrasound molecular imaging concerning the differentiation of benign and malignant ovarian tumors is presented in Figure 2. The safety assessment demonstrated that ultrasound molecular imaging with BR55 was well tolerated by all patients, without any safety concerns noted.

To date, these are the only two papers of ultrasound molecular imaging which described their application in clinical trials. These data indicate that ultrasound molecular imaging with BR55 is clinically feasible and safe, laying the foundation for a new field of ultrasound molecular imaging technology for clinical application in cancer. However, it needs to be pointed out that microbubbles are the intravascular blood flow tracer; ultrasound molecular imaging predominantly targets the specific biomarkers expressed on endothelial cells in the areas of disease, or other vascular targets, such as thrombi or intravascular activated leukocytes attached onto the vessel wall. Although BR55-based ultrasound molecular imaging already shows potential in the differentiation of benign and malignant tumor lesions, some key points have to be emphasized. First, these clinical trials assessed diagnostic accuracy of ultrasound molecular imaging for three kinds of tumors, with only one target receptor. Due to significant variability and heterogeneity of tumors, heterogeneity of target biomarkers expression may also be significant. Therefore, it may be necessary to explore a wide variety of targeted microbubbles and test those microbubbles in a variety of tumors. However, many known tumor biomarkers are associated with tumor cells directly, and might not be present on the luminal surface of endothelium that lines up tumor blood vessels. The micrometer-scale targeted microbubbles are intravascular agents. They are not capable of crossing tumor vessel walls to enter the tumor interstitium; thus, they will not be able to reach and bind with the tumor cell receptors. So, some of the tumor molecular classification information may not be provided by the intravascular targeted microbubble contrast agents. In addition, the KDR-positive ultrasound signals from 


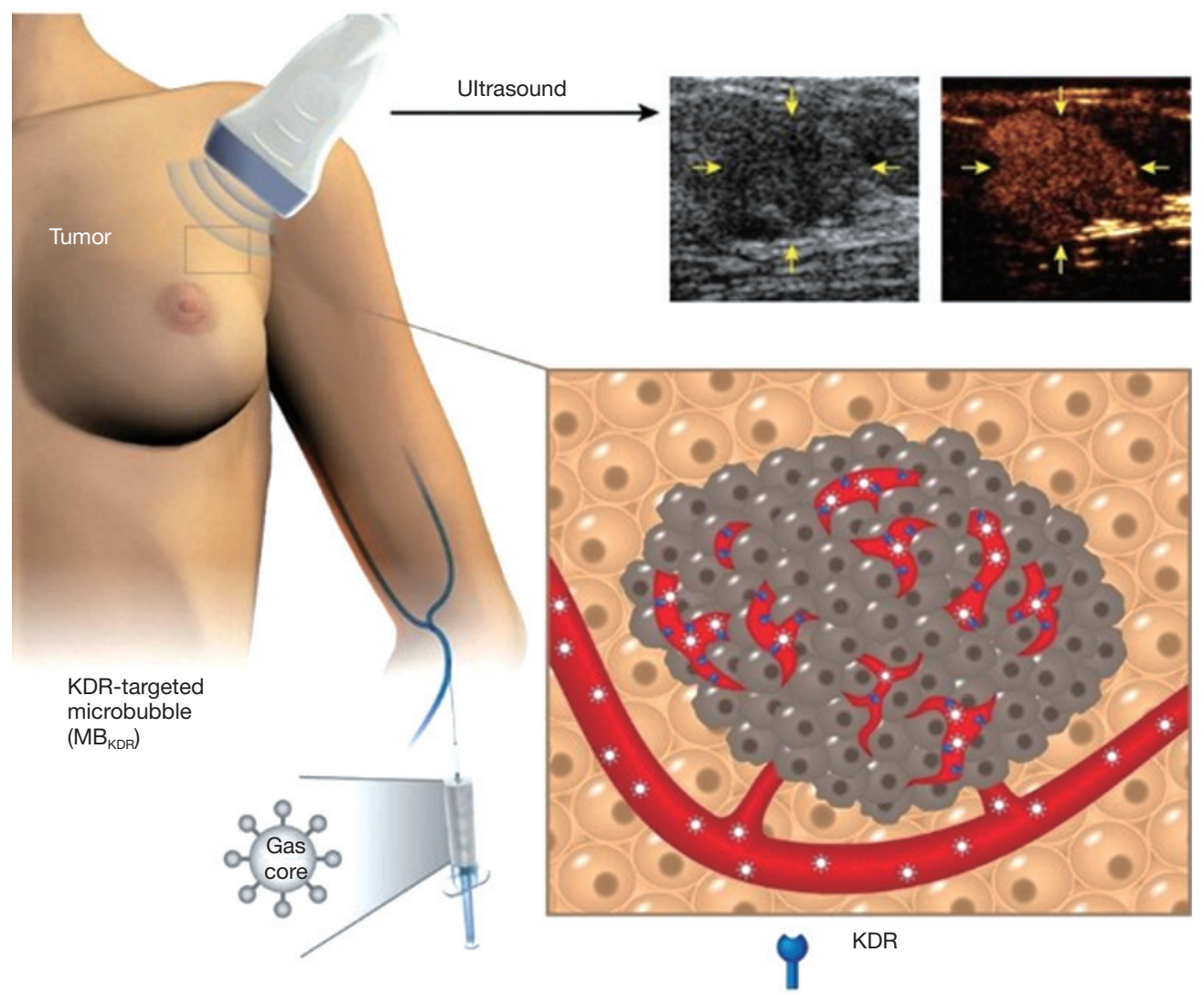

Figure 1 Ultrasound molecular imaging in patients with breast cancer. Targeted contrast microbubbles $\left(\mathrm{MB}_{\mathrm{KDR}}\right)$ which can attach to the breast cancer neovasculature overexpressing kinase insert domain receptor (KDR, shown as a blue receptor) are injected intravenously. The accumulation of $\mathrm{MB}_{\mathrm{KDR}}$ in breast cancer (yellow arrows) can be seen as a strong signal on a contrast mode image (right) using clinical ultrasound equipment. Note that the brightness mode is shown to the left of the contrast mode image for anatomic reference. Reprinted with permission from Copyright 2017 American Society of Clinical Oncology (21).

BR55 molecular imaging trials were still semi-quantitative. A linear relationship between the ultrasound molecular imaging signals and the degree of malignancy of tumors should be confirmed. Other potential artifacts, such as acoustic shadowing, should be avoided, to minimize the influence on diagnostic accuracy.

\section{Challenges and future opportunities}

Microbubble-based ultrasound molecular imaging may have significant potential in early diagnosis. As targeted ultrasound contrast agents improve the sensitivity and specificity of ultrasonic diagnosis, it is the time to extensively explore further clinical applications in more disease types. Some bottlenecks still need to be overcome for the clinical translation of ultrasound molecular imaging, including the development of clinically translatable targeted bubbles and improvement of ultrasound imaging techniques. For instance, antibodies have to be humanized and the linkage strategy through biotin-avidin should be replaced by alternative coupling schemes, to avoid any risk of immune response. Small ligand molecules such as peptides or peptide mimetics can be attached to the shell-forming material (protein or lipid) prior to bubble generation (22). Amalgamation procedure of microbubble generation allows rapid bedside preparation of targeted ultrasound contrast agent particles in sealed sterile vials (23). With proper co-surfactants, this procedure assures efficient transfer of ligand-lipid to the bubble shell, and does not require removal of unincorporated material to achieve efficient targeting in vivo (24).

Targeted adhesion and retention of microbubble contrast 

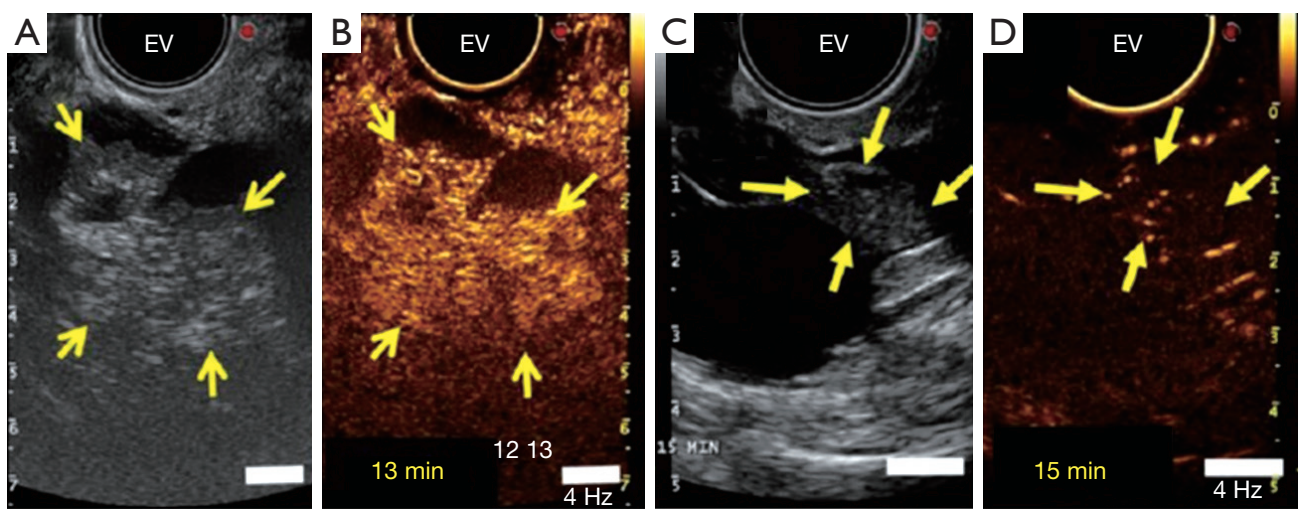

Figure 2 Ultrasound molecular imaging of ovarian lesions with BR55. (A) A transverse endovaginal (EV) low mechanical index reference B-mode ultrasound image of the right ovary in a 50-year-old woman shows a $5.2 \mathrm{~cm}$ cystic and solid lesion (yellow arrows point to solid portion); (B) 13 minutes after intravenous administration of BR55, a strong imaging signal is seen in the solid portion of the lesion (yellow arrows) on contrast mode ultrasound molecular image; (C) a transverse endovaginal low mechanical index reference B-mode ultrasound image of the right ovary shows a $4.8 \mathrm{~cm}$ cystic and solid ovarian lesion (yellow arrows point to solid portion) in a 65 -year-old woman; (D) 15 minutes after intravenous administration of BR55, only a minimal background signal is seen in the $1.3 \mathrm{~cm}$ solid part of the lesion (yellow arrows) on contrast mode ultrasound molecular imaging. Reprinted with permission from Copyright 2017 American Society of Clinical Oncology (21).

agent particles to the receptor surface in flow depends on the fluid shear stress as well as on the microbubble and substrate molecular composition and site surface densities. Flow shear is a significant challenge for adherent microbubbles in fast flow. To improve the efficacy of microbubble targeting, novel strategies may be used to advance their ability to bind to the specific molecular biomarkers expressed on the vessel wall. One approach is to develop dual-targeted contrast agents that could be useful for microbubble binding at physiologically relevant shear stresses (25). Another strategy is to apply acoustic radiation force. The radiation force produced by ultrasound at low pressures and long pulse lengths translates acoustically active particles along the axis of the ultrasound beam without disruption of the agent; i.e., forces particles from the center of the vessel to the vessel wall. This procedure assists the specific adhesion and accumulation of targeted contrast agents to the respective target receptors in fast flow (26). If bubbles are brought to a vessel wall that does not have exposed target biomarker receptors, then upon cessation of acoustic radiation force application, microbubbles will be detached from the target surface and removed with the flow (27). Another novel approach is based on the ability of modern ultrasound to distinguish between adherent microbubbles and microbubbles that move with the flow of blood (28). Application of ultrafast frame rate ultrasound imaging was proposed almost a decade ago; this technique should be capable of further improvement of microbubble detection sensitivity, spatial resolution and diagnostic accuracy (29). Ultra-low dose imaging of VEGFR2-targeted bubbles in the tumor has already been investigated in a murine tumor model (30).

To bypass the vessel wall barrier of microbubble-based contrast agents and to reach into the tumor tissue, submicron nanoparticle ultrasound contrast agents need to be applied; these materials are at the earlier preclinical research stage. These can be either superheated liquid perfluorocarbon nanodroplets that would become gas bubbles upon ultrasound-triggered conversion (31), or gas-carrying nanoparticles, such as bacterial gas vesicles (32), polymer/ lipid nanobubbles (33), or gas-stabilizing nanocones (34). In all of these cases, particle design ensures enhanced stability and minimizes gas loss, when compared with microbubbles. The targeting of these particles directly to tumor cell-specific biomarkers looks feasible, and targeted adhesion will not be constrained by blood flow. Unfortunately, due to the low volume of entrapped gas, detection sensitivity of nanobubbles by ultrasound may require further improvement before it can reach the clinical application stage. 


\section{Conclusions}

Ultrasound molecular imaging is ready to prove its great potential in clinical application. The results of the initial BR5 5 clinical trials represent exciting steps towards the capability to distinguish benign and malignant tumors non-invasively. With the rapid development of material science, chemistry, acoustical physics, data processing, and multi-disciplinary device engineering, we can expect that ultrasound molecular imaging will find broad clinical application in diagnostics, assessment of therapy or imageguided interventions.

\section{Acknowledgements}

Funding: The authors gratefully acknowledge the support of this research by the Shenzhen Science and Technology Innovation Committee (Grant No. JCYJ20170307165254568, JCYJ20170413100222613), and the National Nature Science Foundation of China (Grant No. 81571701, 81530056, 81727805). AL Klibanov is supported in part via NIH R01 EB023055, awarded by the National Institute of Biomedical Imaging and Bioengineering of the National Institutes of Health. The content of this publication is solely the responsibility of the authors and does not necessarily represent the official views of the National Institutes of Health.

\section{Footnote}

Conflicts of Interest: AL Klibanov is a co-founder and minority shareholder of Targeson, a startup in the area of preclinical targeted imaging of microbubbles. He also declares an R43/44 NIH grant subcontract to the University of Virginia laboratory from SoundPipe Therapeutics, and research support from AstraZeneca to UVA CVRC.

\section{References}

1. Gerhard R, Costa JL, Schmitt F. Benign and malignant apocrine lesions of the breast. Expert Rev Anticancer Ther 2012;12:215-21.

2. Guo R, Lu G, Qin B, Fei B. Ultrasound imaging technologies for breast cancer detection and management: a review. Ultrasound Med Biol 2018;44:37-70.

3. Soler M, Dominguez E, Lucas X, Novellas R, GomesCoelho KV, Espada Y, Agut A. Comparison between ultrasonographic findings of benign and malignant canine mammary gland tumours using B-mode, colour Doppler, power Doppler and spectral Doppler. Res Vet Sci 2016;107:141-6.

4. Jiang ZH, Li KT, Tian JW, Ren M. An overview of the development and application of the sonographic scoring system: differentiation of malignant from benign ovarian tumors. Arch Gynecol Obstet 2016;293:303-10.

5. Lecler A, Boucenna M, Lafitte F, Koskas P, Nau E, Jacomet PV, Galatoire O, Morax S, Putterman M, Mann F, Héran F, Sadik JC, Picard H, Bergès O. Usefulness of colour Doppler flow imaging in the management of lacrimal gland lesions. Eur Radiol 2017;27:779-89.

6. Faingold R, Cassia G, Morneault L, Saint-Martin C, Sant'Anna G. Basal ganglia perfusion using dynamic color Doppler sonography in infants with hypoxic ischemic encephalopathy receiving therapeutic hypothermia: a pilot study. Quant Imaging Med Surg 2016;6:510-4.

7. David E, Cantisani V, De Vincentiis M, Sidhu PS, Greco A, Tombolini M, Drudi FM, Messineo D, Gigli S, Rubini A, Fresilli D, Ferrari D, Flammia F, D'Ambrosio F. Contrast-enhanced ultrasound in the evaluation of parotid gland lesions: an update of the literature. Ultrasound 2016;24:104-10.

8. Ho SS. Current status of carotid ultrasound in atherosclerosis. Quant Imaging Med Surg 2016;6:285-96.

9. Ophir J, Cespedes I, Ponnekanti H, Yazdi Y, Li X. Elastography: a quantitative method for imaging the elasticity of biological tissues. Ultrason Imaging 1991;13:111-34.

10. Rago T, Santini F, Scutari M, Pinchera A, Vitti P: Elastography: new developments in ultrasound for predicting malignancy in thyroid nodules. J Clin Endocrinol Metab 2007;92:2917-22.

11. Zhi H, Ou B, Luo BM, Feng X, Wen YL, Yang HY: Comparison of ultrasound elastography, mammography, and sonography in the diagnosis of solid breast lesions. J Ultrasound Med 2007;26:807-15.

12. Zhan J, Jin JM, Diao XH, Chen Y. Acoustic radiation force impulse imaging (ARFI) for differentiation of benign and malignant thyroid nodules--A meta-analysis. Eur J Radiol 2015;84:2181-6.

13. Rehman H, Raza S, Aziz S, Ahmad AM, Tahir S. Diagnostic Accuracy of Sonoelastography in the noninvasive diagnosis of malignant breast cancer compared to histopathology as a gold standard. J Coll Physicians Surg Pak 2017;27:267-70.

14. Zlitni A, Gambhir SS. Molecular imaging agents for ultrasound. Curr Opin Chem Biol 2018;45:113-20. 
15. Güvener N, Appold L, de Lorenzi F, Golombek SK, Rizzo LY, Lammers T, Kiessling F. Recent advances in ultrasound-based diagnosis and therapy with micro- and nanometer-sized formulations. Methods 2017;130:4-13.

16. Villanueva FS, Wagner WR. Ultrasound molecular imaging of cardiovascular disease. Nat Clin Pract Cardiovasc Med 2008;5 Suppl 2:S26-32.

17. Leong-Poi H. Molecular imaging using contrast-enhanced ultrasound: evaluation of angiogenesis and cell therapy. Cardiovasc Res 2009;84:190-200.

18. Lindner JR. Contrast ultrasound molecular imaging of inflammation in cardiovascular disease. Cardiovasc Res 2009;84:182-9.

19. Deshpande N, Pysz MA, Willmann JK. Molecular ultrasound assessment of tumor angiogenesis. Angiogenesis 2010;13:175-88.

20. Smeenge M, Tranquart F, Mannaerts CK, de Reijke TM, van de Vijver MJ, Laguna MP, Pochon S, de la Rosette JJMCH, Wijkstra H. First-in-human ultrasound molecular imaging with a VEGFR2-specific ultrasound molecular contrast agent (BR55) in prostate cancer: a safety and feasibility pilot study. Invest Radiol 2017;52:419-27.

21. Willmann JK, Bonomo L, Carla TA, Rinaldi P, Rindi G, Valluru KS, Petrone G, Martini M, Lutz AM, Gambhir SS: Ultrasound molecular imaging with BR55 in patients with breast and ovarian lesions: first-in-human results. J Clin Oncol 2017;35:2133-40.

22. Yan F, Xu X, Chen Y, Deng Z, Liu H, Xu J, Zhou J, Tan G, Wu J, Zheng H. A lipopeptide-based $\alpha v \beta 3$ integrintargeted ultrasound contrast agent for molecular imaging of tumor angiogenesis. Ultrasound Med Biol 2015;41:2765-73.

23. Unger EC, Fritz TA, Matsunaga T, Ramaswami VR, Yellowhair D, Wu G. Method of preparing gas and gaseous precursor-filled microspheres. US Patent 5585112, Dec. 17, 1996. Available online: https://patents.google.com/ patent/US5853752

24. Unnikrishnan S, Du Z, Diakova GB, Klibanov AL. Formation of microbubbles for targeted ultrasound contrast imaging: practical translation considerations. Langmuir 2018. doi: 10.1021/acs.langmuir.8b03551. [Epub ahead of print].

Cite this article as: Yan F, Song Z, Du M, Klibanov AL. Ultrasound molecular imaging for differentiation of benign and malignant tumors in patients. Quant Imaging Med Surg 2018;8(11):1078-1083. doi: 10.21037/qims.2018.12.08
25. Ferrante EA, Pickard JE, Rychak J, Klibanov A, Ley K. Dual targeting improves microbubble contrast agent adhesion to VCAM-1 and P-selectin under flow. J Control Release 2009;140:100-7.

26. Rychak JJ, Klibanov AL, Hossack JA. Acoustic radiation force enhances targeted delivery of ultrasound contrast microbubbles: in vitro verification. IEEE Trans Ultrason Ferroelectr Freq Control 2005;52:421-33.

27. Wang S, Hossack JA, Klibanov AL, Mauldin FW Jr. Binding dynamics of targeted microbubbles in response to modulated acoustic radiation force. Phys Med Biol 2014;59:465-84.

28. Herbst EB, Unnikrishnan S, Wang S, Klibanov AL, Hossack JA, Mauldin FW Jr. The use of acoustic radiation force decorrelation-weighted pulse inversion for enhanced ultrasound contrast imaging. Invest Radiol 2017;52:95-102.

29. Couture O, Bannouf S, Montaldo G, Aubry JF, Fink M, Tanter M. Ultrafast imaging of ultrasound contrast agents. Ultrasound Med Biol 2009;35:1908-16.

30. Wang S, Herbst EB, Mauldin FW Jr, Diakova GB, Klibanov AL, Hossack JA Ultra-low-dose ultrasound molecular imaging for the detection of angiogenesis in a mouse murine tumor model: how little can we see? Invest Radiol 2016;51:758-66.

31. Hadinger KP, Marshalek JP, Sheeran PS, Dayton PA, Matsunaga TO. Optimization of phase-change contrast agents for targeting mda-mb-231 breast cancer cells. Ultrasound Med Biol 2018;44:2728-38.

32. Lakshmanan A, Lu GJ, Farhadi A, Nety SP, Kunth M, Lee-Gosselin A, Maresca D, Bourdeau RW, Yin M, Yan J, Witte C, Malounda D, Foster FS, Schröder L, Shapiro MG. Preparation of biogenic gas vesicle nanostructures for use as contrast agents for ultrasound and MRI. Nat Protoc 2017;12:2050-80.

33. Gao Y, Hernandez C, Yuan HX, Lilly J, Kota P, Zhou $\mathrm{H}, \mathrm{Wu} \mathrm{H}$, Exner AA. Ultrasound molecular imaging of ovarian cancer with CA-125 targeted nanobubble contrast agents. Nanomedicine 2017;13:2159-68.

34. Mannaris C, Teo BM, Seth A, Bau L, Coussios C, Stride E. Gas-stabilizing gold nanocones for acoustically mediated drug delivery. Adv Healthc Mater 2018;7:e1800184. 